\title{
Construcción de una guía de densidad para Pinus cooperi var. ornelasi con base en la clase de copa en Durango, México
}

\author{
Marco A. Márquez-Linares ${ }^{1}$ \\ Rebeca Alvarez-Zagoya ${ }^{1}$
}

\begin{abstract}
RESUMEN
El presente estudio consistió en la elaboración de una guía para el manejo de la densidad de Pinus cooperi var ornelasi en el estado de Durango, México. Se utilizó una metodología basada en la evaluación de la densidad en que se encontraron árboles de diferentes clases de copa. La línea de cierre de copas se alcanzó a un índice de densidad de Reineke, con un diámetro de referencia de $25 \mathrm{~cm}$, de 310.31, la línea de árboles dominantes a un IDRR de 658.62, los intermedios a 720.18 y los suprimidos a un IDRR de 1,007.05. Estas líneas determinan posibles rangos de densidad en los cuales un rodal de Pinus cooperi de igual edad puede ser manejado. La metodología utilizada en el presente estudio ofreció resultados similares a los encontrados para otras especies de pinos en México.
\end{abstract}

\section{PALABRAS CLAVE}

Diagrama de manejo de la densidad, Pinus cooperi, Durango, competencia de copas, densidad forestal.

\begin{abstract}
The objective of the present study is to construct a density management diagram for Pinus cooperi var ornelasi stands in the state of Durango, México. The resulting diagram, based on different crow $n$ classes, was constructed for the existing even-aged trees. The line of crow $n$ closure reached a Reineke stand density index of 310.31 , the line for dominant crown trees reached 658.62, the line for intermediate crown trees was 720.18, and the line for suppressed crow $n$ trees was 1007.05 SDi. These lines indicate possible density ranges w ithin which a even-aged Pinus cooperi stand could be managed. The methodology used yielded results similar to those found for other pine species in Mexico.
\end{abstract}

\section{KEY WORDS}

Forest density, Pinus cooperi, Durango, density management diagram, crown competition.

$1 \quad$ CIIDIR-IPN U. Dgo. Negrete 312 Ote. 34000 Durango, Dgo. Tel. y Fax (18) 114437.

Manuscrito recibido para su publicación el 5 de J unio de 1995 


\section{INTRODUCCION}

Los diagramas de manejo de la densidad constituyen una herramienta valiosa para la planeación de regímenes de tratamientos silvícolas que garanticen una óptima ocupación del sitio de acuerdo con los objetivos de producción propuestos para éste. En México se han construido guías de manejo de la densidad para Pinus patula por Becerra, 1986, para Pinus hartwegii por Zepeda y Villareal, 1987 y para P. pseudostrobus por Aguirre et al., 1994. En Norteamérica se encuentran los trabajos de Drew y Flewelling, 1979 para Pseudostsuga menziesii, Flewelling y Drew, 1985 para P. contorta y McCarter y Long, 1986 para P. douglasiana. Esto estudios han confirmado que existe una relación estrecha entre el tamaño de lo individuos y su número por unidad de superficie en diferentes situaciones competitivas. En la literatura ecológica esta relación fue originalmente referida como "principio de autoaclareo" (Yoda y Kira, 1963) y más recientemente como "relaciones interespecíficas de tamaño densidad" (Weller, 1989). Esta relación fundamental, independiente de la edad y de la calidad del sitio, ofrece una base excelente de la cual se desprende el conocimiento de las interacciones competitivas entre los individuos de una población.

En el estado de Durango son escasos los conocimientos cuantitativos sobre las densidades adecuadas para optimizar determinados objetivos de producción en especies locales. Por lo anterior, el objetivo del presente trabajo es conocer los rangos de densidad dentro de los cuales se desarrollan rodales coetáneos de Pinus cooperi var. ornelasi Blanco y expresar estos mediante los diagramas de área basal propuestos por Gingrich, 1967.
La metodología utilizada en el presente trabajo difiere de las utilizadas hasta ahora, en cuanto a la determinación de los niveles de densidad en los que se da la ocupación plena y la máxima densidad que puede soportar el sitio. Mientras que tradicionalmente se utilizan los valores de densidad a nivel rodal en sitios bien ocupados $y$ sobreocupados, en el presente estudio se utiliza un enfoque de árboles individuales, evaluando la densidad en que se encuentran árboles de diferentes clases de copa. Esta metodología aporta datos interesantes en cuanto a los niveles de densidad en que se manifiestan los árboles libres, dominantes, intermedios y suprimidos.

\section{Area de estudio}

El estudio se llevó a cabo en el Municipio de San Dimas, Dgo., en el predio particular "La Quinta" y sus alrededores, ubicado a $160 \mathrm{~km}$ al oeste de la ciudad de Durango, a los $24^{\circ} 21^{\prime}$ de latitud norte y $105^{\circ} 30^{\prime}$ longitud oeste (Fig. 1). La topografía de este lugar está conformada por mesetas, llanos y lomeríos de pendientes suaves a medias. La altitud del predio varía entre 2,400 y $2,500 \mathrm{msnm}$.

De acuerdo a Köppen modificado por García, 1982, el clima corresponde a C(e) (W2) semifrío subhúmedo con lluvias en verano, temperatura media anual de $11.5^{\circ} \mathrm{C}$. La precipitación media anual es de $851 \mathrm{~mm}$ y la precipitación del mes más seco es menor a $40 \mathrm{~mm}$. De acuerdo a la clasificación FAO-UNESCO modificada por DETENAL, los suelos de esta región caen dentro de las categorías siguientes: litosol, regosol eútrico y cambisol eútrico.

La vegetación corresponde a bosque de pino-encino con presencia de otras latifoliadas como son el madroño (Arbutus madrensis y $A$. tesselata). Las especies de encinos de mayor abundancia en el sitio 


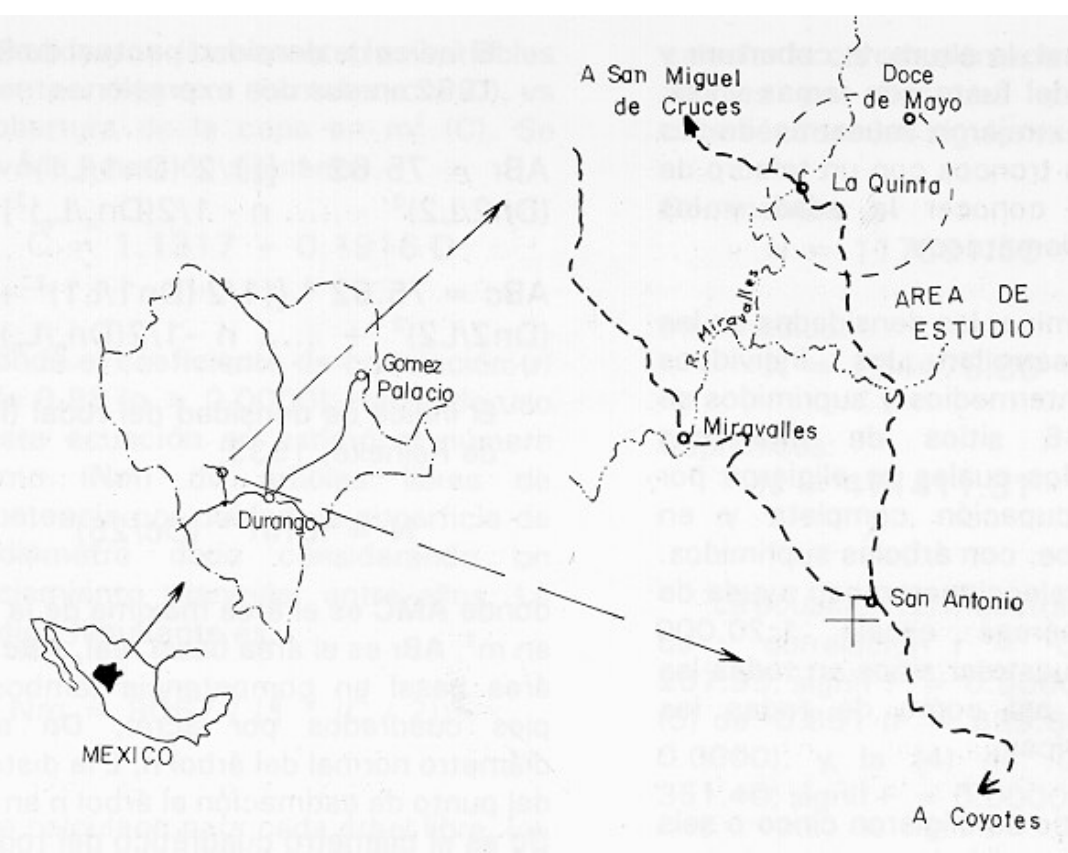

Figura 1. Ubicación del área de estudio

son Quercus sideroxyla Humb. \& Bonpl. y Q. durifolia V. Seem. y las de pino son Pinus cooperi Ornelasi, P. durangensis Schl. y P. leiophylla Schl. \& Cham. El estrato herbáceo está formado principalmente por pastos (Bouteloua spp) en áreas abiertas, y de manzanilla (Arcthosthaphilos pungens HBK.) en sitios perturbados.

\section{Descripción de la especie}

Pinus cooperi var. ornelasi Blanco, es un árbol que puede alcanzar 35 metros de altura con una copa redonda y densa, ramas inclinadas hacia abajo y corteza de color café cenizo (Martínez, 1992). Ocurre en la Sierra Madre Occidental desde la parte sur del estado de Chihuahua hasta la parte centro del estado de Durango, en un rango altitudinal que varía de los 2,000 a 2,800 msnm. Se desarrolla preferentemente en sitios de pendientes suaves o zenitales, generalmente en las faldas de lo cerros y a orillas de los llanos. Conforme aumenta la pendiente en los lomeríos, P. cooperi es substituído por P. teocote, P. leiophylla y P. durangensis. Comúnmente se encuentra formando masas puras, sin embargo, en ocasiones se encuentra asociado con Quercus sideroxyla, J unipperus deppeana y en ocasiones con Pinus leiophylla (Márquez, 1994).

\section{METODOLOGÍA}

La determinación de la línea de cierre de copas se realizó mediante la metodología propuesta por Krajiceck y Brinkman, 1961. Para este efecto se seleccionaron 58 árboles que presentaran diámetros normales de 10 a $60 \mathrm{~cm}$, y que tuvieran la copa libre de competencia con otros árboles. En cada árbol se consideraron las variables siguientes: el 
diámetro normal, la altura, la cobertura y la proporción del fuste con ramas vivas. También se extrajeron muestras de los núcleos de los troncos con un taladro de Pressler para conocer la edad y los incrementos diamétricos.

Para determinar las densidades en las que se desarrollan los individuos dominantes, intermedios y suprimidos se levantaron 46 sitios de diferentes dimensiones, los cuales se eligieron por tener una ocupación completa y en algunos de ellos, con árboles suprimidos. Los sitios se seleccionaron con ayuda de fotografías aéreas escala 1:20,000 procurando muestrear sitios en todas las exposiciones, así como de todas las clases diamétricas.

En cada sitio se eligieron cinco o seis árboles, más los árboles que mostraran sobreposición de copas en torno a los primeros. Se elaboró en cada sitio un mapa de la ubicación de todos los árboles por medio de triangulaciones. En todos los árboles se tomaron los mismos datos que en los árboles libres y se clasificaron sus copas en dominantes (aquellos con copa sobresaliente por encima del nivel medio del dosel), intermedios (aquellos con la altura media del dosel) y suprimidos (aquellos con altura por abajo del nivel medio del dosel). En total se muestrearon 114 árboles de todas las categorías diamétricas y 282 árboles vecinos.

Se determinó la densidad a que están sujetos los árboles objetivo por medio de cuatro índices:

El índice de interferencia de copas de Krajicek y Brinkman, 1962, que tiene la siguiente expresión matemática

$$
\mathrm{FCC}=\left(\mathrm{AMC}_{\mathrm{i}}\right) / \text { área } * 100
$$

El índice de densidad puntual de Spurr, 1962 en sus dos expresiones

$\mathrm{ABr}=75.62 *\left\{\left[1 / 2(\mathrm{Dn} 1 / \mathrm{L} 1)^{2}-3 / 2\right.\right.$ $\left.\left.(\mathrm{Dn} 2 / \mathrm{L} 2)^{2}-\ldots . \mathrm{n}-1 / 2\left(\mathrm{Dn} n / L_{n}\right)^{2}\right] / \mathrm{n}\right\}$

$$
A B C=75.62 *\left\{\left[1 / 2(D n 1 / L 1)^{2}+3 / 2\right.\right.
$$$$
\left.\left.(\mathrm{Dn} 2 / \mathrm{L} 2)^{2}+\ldots . . \mathrm{n}-1 / 2\left(\mathrm{Dn_{n }} / \mathrm{Ln}_{\mathrm{n}}\right)^{2}\right] / \mathrm{n}\right\}
$$

El índice de densidad del rodal (IDRR) de Reineke, 1933:

$$
N=\operatorname{IDRR} *(D c / 25)^{b}
$$

donde $\mathbf{A M C}$ es el área máxima de la copa en $\mathrm{m}^{2}, \mathbf{A B r}$ es el área basal real, $\mathbf{A B c}$ es el área basal en competencia (ambos, en pies cuadrados por acre), Dn es el diámetro normal del árbol $\mathbf{n}$, L la distancia del punto de estimación al árbol $\mathbf{n}$ en pies, Dc es el diámetro cuadrático del rodal en centímetros y $\mathbf{N}$ es el número de individuos por hectárea.

El índice de densidad puntual real de Spurr se tomó como base para determinar la densidad en términos de $\mathrm{m}^{2} /$ ha en la que se encuentra cada árbol objetivo. El número de árboles por hectárea se determinó utilizando el diámetro normal de cada árbol y el área basal real. La base de datos consistió en la clase de copa, el diámetro normal, y el área basal. Con estos datos se determinaron las ecuaciones de regresión lineal simple para cada clase de copa. Las ecuaciones así obtenidas se compararon mediante un análisis de covarianza, un análisis de comparación entre las pendientes y entre las elevaciones de acuerdo a la metodología propuesta por Zar, 1984.

\section{RESULTADOS}

Los datos obtenidos de los árboles en posición libre de competencia, se 
correlacionaron linealmente las variables siguientes: diámetro normal en $\mathrm{cm}$ (D), vs la cobertura de la copa en $\mathrm{m}^{2}$ (C). Se obtuvo la ecuación siguiente:

$$
C=1.1317+0.1916 \mathrm{D}
$$

en donde el coeficiente de correlación ( $\mathbf{r}$ ) fue de 0.98 ( $p>0.0000)$. Haciendo uso de ésta ecuación se estimó el número máximo ( $\mathbf{N m})$ de árboles libres de competencia por unidad de superficie de un diámetro dado considerando un espaciamiento triangular entre ellos. La ecuación resultante es:

$$
\mathrm{Nm}=9067 /(\pi *(\mathrm{C} / 2))^{2}
$$

Se calculaon para cada árbol libre, los valores del logaritmo del número de individuos por hectárea (N) y el logaritmo del diámetro. Con estos datos se obtuvo la línea de cierre de copa descrita por la siguiente ecuación :

$$
N=29505.30 d-1.4271
$$

El modelo (1) tiene un coeficiente de correlación $\mathbf{r}=0.98732(\mathrm{~F}=4283.85$; signif $F=0.0000)$. Con la estimación del área basal real de Spurr para cada árbol individual, se calculó el número de árboles por hectárea con un diámetro similar al del árbol objetivo mediante la siguiente ecuación:

$$
N=A B /\left(\pi *(D n / 2000)^{2}\right)
$$

Una vez divididos los datos según la posición de la copa del árbol, se emplearon las ecuaciones anteriores para calcular la correlación entre el número de árboles por hectárea y el diámetro, obteniéndose las siguientes ecuaciones:

Dominantes:

$$
\mathrm{N}=117381.59 * \mathrm{D}^{-1.6102}
$$

Intermedios:

$$
N=234476.86 * D^{-1.7974}
$$

Suprimidos:

$$
N=471411.31 * D^{-1.19102}
$$

La ecuación (2) muestra un coeficiente de correlación $\mathbf{r}=-0.8798,(\mathrm{~F}=$ 207.93; signif $F=0.0000)$, la ecuación (3) de -0.851 ( $F=639.66$; signif $F=0.0000$ ), y la (4) de -0.7196 $(F=351.40 ;$ signif $F=0.0000)$.

El análisis de covarianza indica que existen diferencias significativas entre los coeficientes de regresión $(F=24.11$,

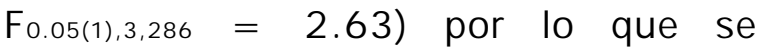
procedió a realizar una comparación entre los coeficientes de las líneas (Tabla 1). En cuanto a las elevaciones de las líneas que tienen estadísticamente la misma pendiente, se aplicó una comparación entre elevaciones (Tabla 2).

La Tabla 3 muestra el crecimiento promedio observado en la categoría de 10 a $19.9 \mathrm{~cm}$ de diámetro así como el porcentaje de copa viva de los árboles de las diferentes clases de copa, mientras que la Tabla 4 compara los resultados del presente estudio por los obtenidos por Zepeda y Villarreal, 1987 para Pinus hartwegii, Aguirre et al., 1994 , para P. pseudostrobus y los citados por Long, 1985, para P. ponderosa y P. contorta. 
Tabla 1. Comparación entre los coeficientes de las ecuaciones de regresión de las diferentes clases de copa

\begin{tabular}{lcccc} 
ECUACIONES & $\mathbf{V}$ & “t” & “t”.05(2) & $\mathbf{H}_{\mathbf{0}}: \mathbf{b}_{\mathbf{1}}=\mathbf{b}_{\mathbf{2}}$ \\
\hline $\mathrm{L}(1)$ vs $\mathrm{D}(2)$ & 140 & 1.595 & 1.976 & aceptada \\
$\mathrm{L}(1)$ vs I(3) & 148 & 2.355 & 1.976 & rechazada \\
$\mathrm{L}(1)$ vs S(4) & 108 & 2.341 & 1.982 & rechazada \\
$\mathrm{D}(2)$ vs I(3) & 178 & 1.4176 & 1.973 & aceptada \\
$\mathrm{D}(2)$ vs S(4) & 138 & 1.714 & 1.977 & aceptada \\
$\mathrm{I}(3)$ vs S(4) & 146 & 0.508 & 1.976 & aceptada \\
\hline
\end{tabular}

D: dominantes L: libres I: intermedios S: suprimidos

Tabla 2. Comparación entre las elevaciones de las ecuaciones de regresión de las diferentes clases de copa

\begin{tabular}{lcccc} 
ECUACIONES & $\mathbf{V}$ & “t” & “t”.05(2) & $\mathbf{H}_{\mathbf{0}}: \mathbf{a}_{\mathbf{1}}=\mathbf{a}_{\mathbf{2}}$ \\
\hline $\mathrm{L}(1)$ vS $\mathrm{D}(2)$ & 141 & 3.012 & 1.977 & rechazada \\
$\mathrm{D}(2)$ vs I(3) & 179 & 3.583 & 1.973 & rechazada \\
$\mathrm{D}(2)$ vs S(4) & 139 & 6.584 & 1.977 & rechazada \\
I(3) vs S(4) & 147 & 3.431 & 1.976 & rechazada \\
\hline
\end{tabular}

D: dominantes L: libres I: intermedios S: suprimidos

Tabla 3. Porcentaje de copa viva e incremento corriente anual observado en los árboles de diferentes clases de copa

\begin{tabular}{lcc} 
CLASES DE COPA & $\begin{array}{c}\text { PORCENTAJ E DE } \\
\text { COPA VIVA }\end{array}$ & $\begin{array}{c}\text { ICA Promedio } \\
\mathbf{( 1 0 - 1 9 . 9} \text { Dn) }\end{array}$ \\
\hline Libres & $81.9 \pm 7.4$ & $0.23 \pm 0.09$ \\
Dominantes & $43.0 \pm 1.5$ & $0.28 \pm 0.02$ \\
Intermedios & $34.3 \pm 1.3$ & $0.18 \pm 0.02$ \\
Suprimidos & $23.5 \pm 2.5$ & $0.15 \pm 0.03$ \\
\hline
\end{tabular}


Tabla 4. Comparación entre los índices de rodal de Reineke $(\mathrm{d}=25 \mathrm{~cm})$ de Pinus cooperi y otras especies de pinos

\begin{tabular}{|c|c|c|c|c|}
\hline ESPECIE & FCCC & RAA & $\begin{array}{l}\text { LIMITE BAJ O DE } \\
\text { OCUPACION } \\
\text { TOTAL }\end{array}$ & $\begin{array}{l}\text { LIMITE BAJ O DE } \\
\text { AUTOACLAREO }\end{array}$ \\
\hline Pinus cooperi & 310.3 & $658.6(\mathrm{D}$ & $720.2(\mathrm{I})$ & $1007.1(\mathrm{~S})$ \\
\hline P. hartw egii ${ }^{1}$ & 496 & 766 & & \\
\hline P. pseudostrobus ${ }^{2}$ & 259 & 873 & & \\
\hline P. ponderosa ${ }^{3}$ & 510 & & 720 & 1225 \\
\hline P. contorta ${ }^{3}$ & 425 & & 600 & 1020 \\
\hline $\begin{array}{l}1 \text { Zepeda y Villa } \\
2 \text { Aguirre et al., } \\
3 \text { Long, } 1985\end{array}$ & 987 & $\begin{array}{l}\text { D: } \\
\text { I: } \\
\text { S: }\end{array}$ & $\begin{array}{l}\text { dominantes } \\
\text { intermedios } \\
\text { suprimidos }\end{array}$ & \\
\hline
\end{tabular}

\section{DISCUSION}

El análisis estadístico efectuado demuestra que las cuatro ecuaciones de regresión lineal calculadas para los árboles libres, dominantes, intermedios y suprimidos, son significativamente diferentes ya sea en su pendiente y/o en su elevación, por lo que se asume que los árboles de diferente clases de copa se manifiestan en diferentes condiciones de densidad.

El comportamiento esperado de las pendientes de las cuatro regresiones es de -1.5 que corresponde a la propuesta por Yoda, 1963 en el principio de autoaclareo. Este principio propone que en rodales de alta densidad existe una relación de $-3 / 2$ entre el tamaño (volumen, diámetro normal u otro) y el número de individuos por unidad de superficie. Aunque se ha visto que encontrar esta relación en árboles, es más bien una excepción que una regla (Sprugel, 1984, citado en
Weller, 1989), y que la relación puede cambiar con la especie, el sitio, la edad y la tolerancia de la especie a la sombra.

En el caso de los individuos dominantes e intermedios, este comportamiento se acerca a lo esperado por el principio de autoaclareo, ya que la pendiente de estas regresiones se encuentra dentro de los intervalos de confianza, las cuales son de -1.62 \pm 0.2222 y $-1.797 \pm 0.3048$, respectivamente.

En el caso de los individuos libres la relación entre la cobertura y el diámetro normal es muy estrecha pero la pendiente no corresponde a lo esperado. Sin embargo, en los individuos menores a 10 cm de diámetro se observó un comportamiento diferente a los de mayor tamaño en cuanto a la relación entre la cobertura y el diámetro. Probablemente esto se deba a que en dichos individuos aún no se expresa la cobertura de la copa 
plenamente. Si se eliminan de los cálculos a estos árboles, se obtiene la siguiente ecuación:

$$
N=56483.16 * d^{-1.61675}
$$

La pendiente de esta ecuación $\left(r^{2}=\right.$ $0.9982, F=26402$, signif $=0.0000$ ) es prácticamente la misma que la que se obtiene para los individuos dominantes. Esto significa que la relación entre el tamaño y número de individuos por unidad de superficie se mantiene constante en el espacio de densidad comprendido entre los árboles libres y los dominantes variando solamente el grado de densidad en el cual se encuentran (elevación) como se explicará más adelante.

La línea de los individuos libres o de cierre de copas, describe el grado de densidad a partir del cual inicia el desarrollo del rodal. Por debajo de este punto los árboles manifiestan características especiales denominadas efecto "lobo", como son un alto porcentaje de la altura del árbol con ramas vivas por el bajo grado de autopoda, coberturas muy amplias, fustes cónicos, y una altura menor en relación con otros individuos de su misma edad.

Por las razones anteriores, la densidad mínima deseable para el desarrollo de un rodal comercial tendría que ser mayor a la de esta línea para que la competencia interespecífica elimine estos efectos, los cuales no son deseables desde el punto de vista comercial. En el área de estudio, Pinus cooperi junto con $P$. engelmannii, son las únicas especies que se desarrollan en áreas abiertas y manifiestan las características de árboles "lobo". En otras especies como $P$. durangensis, $P$. teocote o P. leiophylla, es raro que se encuentren en esta condición.
En los individuos dominantes se observaron los mayores incrementos diamétricos a nivel de árbol individual (Tabla 3), lo cual es reflejo tanto de la posición de la copa como el porcentaje de altura con ramas vivas que poseen, que en promedio es de $43 \% \pm 1.5$. De acuerdo a Daniel, et al., 1979, dicho porcentaje es característico de individuos vigorosos y que pueden reaccionar positivamente a aclareos. El nivel de densidad al cual se hallaron los individuos dominantes representa el punto en el cual los árboles tienen el espacio de crecimiento mínimo indispensable para desarrollarse plenamente por lo que podría considerarse equivalente a la línea de la relación área-árbol (Chisman y Schumacher, 1940) y al índice de rodal de Reineke,1933. Entonces, el IDRR para Pinus cooperi está dado por:

$$
N=658.6(d / 25)^{-1.6102}
$$

La línea que corresponde a los árboles intermedios, se puede considerar como el límite máximo de densidad deseable para el manejo de rodales de P. Cooperi, ya que los árboles que se encuentran en este nivel de densidad muestran una proporción de copa viva de $34 \% \pm 1.3$, porcentaje que esta ligeramente bajo, según los considerados por Daniel et al., 1979, como óptimos para obtener crecimientos altos en coníferas. Este nivel de densidad puede ser equivalente a la línea de mortandad inminente, es decir el punto a partir del cual empieza a existir mortalidad debido a la competencia interarbórea.

Para los individuos suprimidos la pendiente observada es de -1.910 \pm-0.2048 , la cual se aleja con mucho de lo observado en los individuos libres y dominantes. Esto puede obedecer a dos causas: la escasez de individuos 
suprimidos de diámetros mayores a 25 $\mathrm{cm}$, los cuales fueron eliminados por intervenciones silvícolas anteriores, y a la alta densidad registrada en sitios con árboles de diámetros menores a $5 \mathrm{~cm}$, lo cual, de acuerdo a Westoby, 1984, es uno de los posibles casos donde se pueden hallar sitios con densidades mayores a las esperadas por la línea de autoaclareo. Los dos hechos anteriores pueden ser la causa de la pendiente encontrada en árboles suprimidos no corresponda a la esperada. No obstante, el nivel de densidad al que se encontraron estos árboles es exagerado ya que en este punto todos los árboles serian eliminados por la competencia interespecífica. En los sitios estudiados los árboles suprimidos se encontraron junto a árboles dominantes e intermedios, los cuales generan la supresión en los primeros. Por esto, a nivel rodal, seguramente la línea de autoaclareo se manifiesta a un nivel de densidad menor que el observado en la línea de los suprimidos.

En las ecuaciones de los árboles de diferentes clases de copa se observa una tendencia a disminuir la relación entre el diámetro y la densidad conforme la competencia aumenta. Es decir, la pendiente más alta corresponde a los individuos libres y dominantes (-1.61), los intermedios (-1.79) y finalmente los suprimidos (-1.91). Esta tendencia sugiere que la competencia tiene un efecto importante en la pendiente de la relación tamañodensidad, a partir del punto en el cual la competencia se hace más intensa (intermedios).

El efecto descrito en el párrafo anterior se puede explicar en el sentido de que en los individuos libres y dominantes la copa se expresa plenamente; la diferencia entre ambos, es el porcentaje de la altura con ramas vivas, sin embargo, el requerimiento de espacio se mantiene proporcional en ambos casos porque no se altera la forma de la copa produciéndose líneas paralelas. En los individuos intermedios, al existir un mayor grado de interferencia de copas con los vecinos, la copa ya no se expresa plenamente ya que el crecimiento de las ramas disminuye por el sombreado de los árboles vecinos $\mathrm{y}$, por tanto, se altera la relación espacio-tamaño obteniéndose, por esto, una pendiente mayor. En los individuos suprimidos, la cobertura de las ramas y la proporción de copa viva es relativamente mucho menor (Tabla 4), por lo que la relación espacio-tamaño se encuentra totalmente alterada como se manifiesta en la pendiente de (-1.91).

La figura 2 (a y b) corresponde a un diagrama que relaciona el área basal, el diámetro y el número de árboles por hectárea para determinar los rangos de densidad en que se desarrollan rodales coetáneos de Pinus cooperi. Este tipo de diagramas fue propuesto originalmente por Gingrich, 1967 con la diferencia que en el diagrama de la figura 2 se expresan las líneas de densidad en que se desarrollan los individuos de diferentes clases de copa.

El diagrama se construyó haciendo uso de las ecuaciones 2, 4 y 5 . De la ecuación 3 (intermedios) se utilizó el IDRR a $25 \mathrm{~cm}$ y se utilizó el coeficiente de la ecuación 3 (dominantes), ya que la ecuación 3 se intercepta con la 2 debido a la diferencia de pendientes, lo cual no tiene sentido biológico. Esto es aceptable porque $25 \mathrm{~cm}$ representa aproximadamente la media de la distribución diamétrica del muestreo además de que el valor asignado para la pendiente entra dentro de la banda de confianza de los intermedios, como se explicó anteriormente.

La figura 2 describe tres espacios de crecimiento: el comprendido entre la línea 
de cierre de copas y los individuos dominantes dentro del cual se dan condiciones de crecimiento optimas; el espacio que se da entre los individuos dominantes y los intermedios en el cual se empiezan a manifestar efectos competitivos negativos, el espacio comprendido entre la línea de los intermedios y de los suprimidos (autoaclareo) que debe de evitarse para fines de manejo, ya que el rendimiento disminuye la mortalidad por competencia intraespecífica se manifiesta de manera significativa.

En la Tabla 4 se observan los valores del IDRR de P. cooperi y de otras especies de México y Norteamérica. Las metodologías empleadas para el cálculo de los valores varían de una especie a otra, así como los criterios de espaciamiento. No obstante, se observa que los valores de IDRR obtenidos para las diferentes especies coinciden dentro de un rango de variación que va para la línea de cierre de copas de 259 a 510, para la ocupación óptima (dominantes, RAA y límite bajo de ocupación total) de 600 a 873,y para la límite bajo de autoaclareo de 1,007 a 1,225 . En este contexto, en $P$. cooperi se observan valores relativamente más bajos lo cual puede indicar que esta especie requiere de un mayor espacio para su desarrollo. Lo anterior tiene implicaciones importantes para su manejo, ya que para un mismo objetivo de producción esta especie puede requerir espaciamientos mayores en comparación a otras especies.

Es necesario indicar que los resultados aquí expuestos al ser basados sobre las densidades en árboles individuales reflejan tanto el espacio horizontal disponible para ellos en el momento del muestreo, como el espacio vertical el cual es reflejado en la clase de copa a la cual pertenece cada individuo. En este sentido la metodología aplicada puede tener ventajas para evaluar las necesidades de espacio para obtener rendimientos óptimos.

\section{CONCLUSIONES}

En el presente estudio utilizó una metodología para ubicar las relaciones de tamaño-densidad de Pinus cooperi con base en el análisis de la densidad en que se encuentran árboles individuales de diferentes clases de copa. Esta metodología ofrece una alternativa para evaluar los espacios de crecimiento necesarios para construir diagramas de manejo de la densidad de especies forestales que se desarrollan de manera natural con estructuras regulares $y$ monoespecíficas.

De acuerdo con los resultados, Pinus cooperi es una especie que requiere más espacio para su crecimiento en comparación con otras especies mexicanas, lo cual tiene implicaciones importantes para su manejo. En este sentido, los diagramas de manejo de la densidad permiten tener una referencia de gran utilidad práctica para conocer los espacios de crecimiento requeridos por cada especie para su crecimiento optimo.

\section{AGRADECIMIENTOS}

Se agradece a la Unidad de Conservación y Desarrollo Forestal No. 4. "La Victoria - Miravalles", Dgo. Las facilidades prestadas para llevar acabo el presente estudio y en particular al Sr. Don Alfredo Díaz Barraza. 


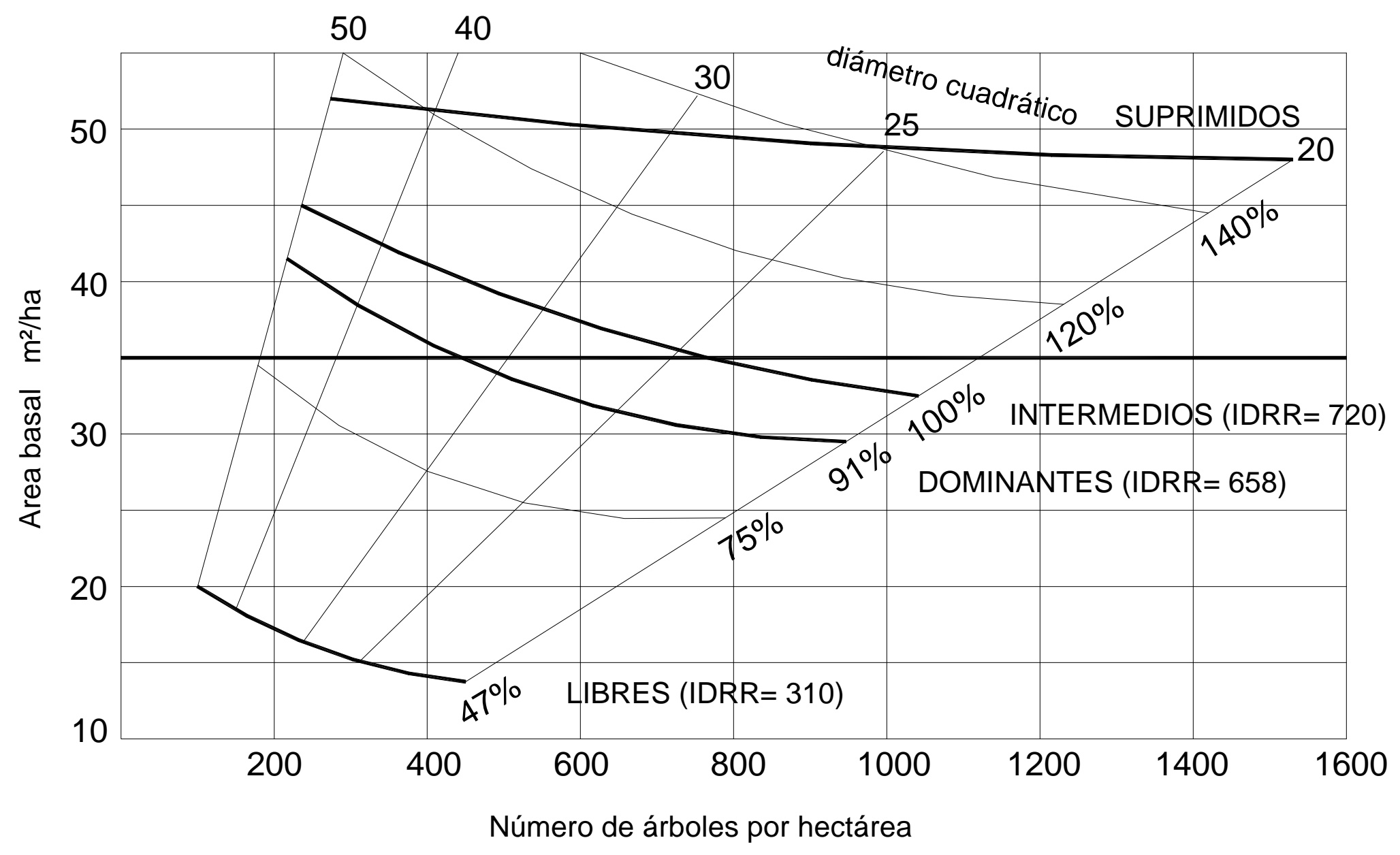

Figura 2a. Diagrama de densidad arbórea de Pinus cooperi var ornelasi en Durango, México 


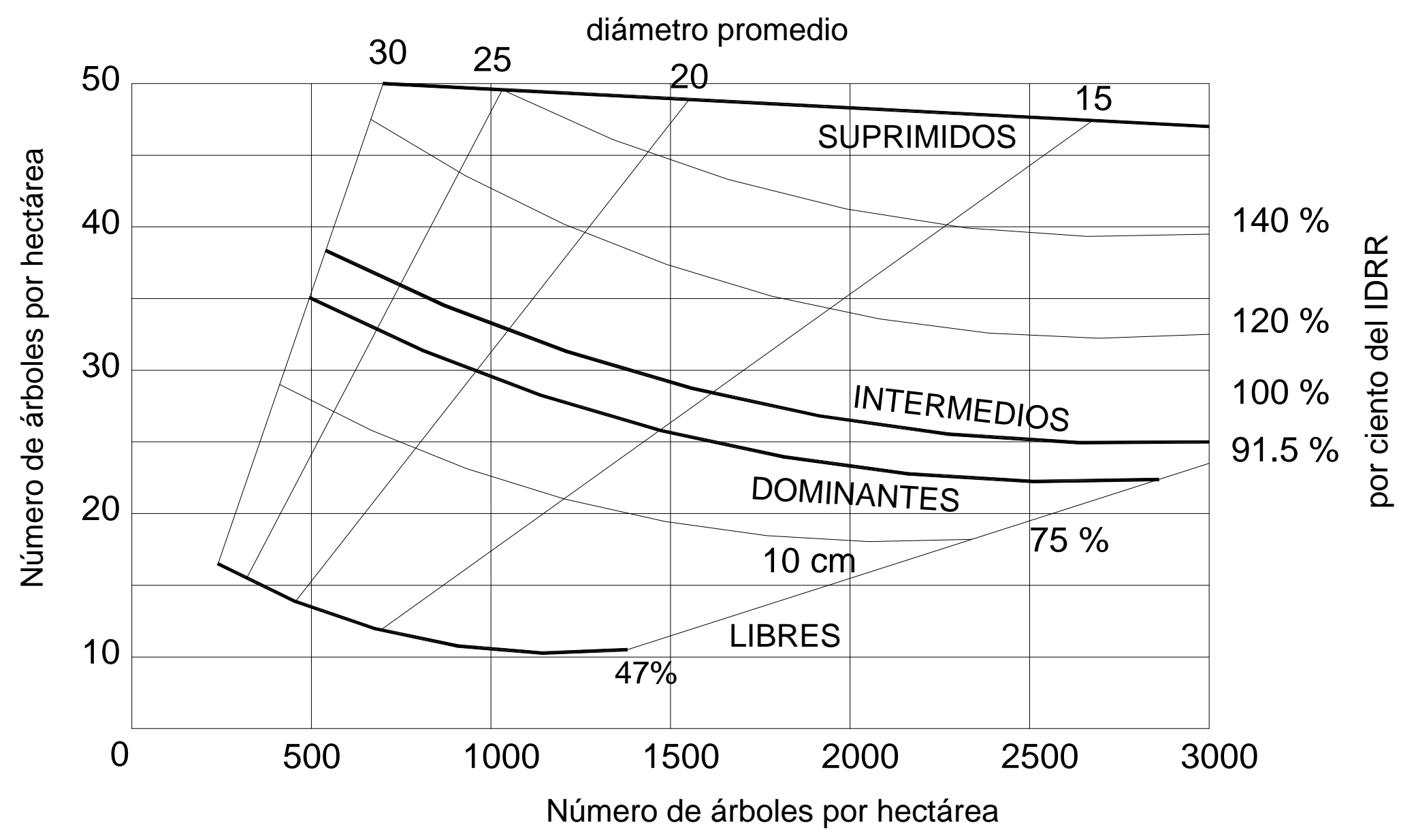

Figura 2a. Diagrama de densidad arbórea de Pinus cooperi var ornelasi en Durango, México 


\section{REFERENCIAS}

Aguirre, C.0.; G. Vega y J. Jiménez. 1994. Guía de densidad para Pinus pseudostrobus Lindl. en Nuevo León. Memorias del Simposio y II Reunión Nacional de Silvicultura y Manejo de Recursos Forestales. p. 43.

Becerra, L. 1986. Determinación de una guía de densidad para Pinus patula Schdl. En Chignahuapan, Zacatlán, Pue. Tesis de M. en Ciencias, Colegio de Postgraduados. Chapingo, Méx. $82 \mathrm{pp}$.

Chisman, H. H. y F. X. Shumacher. 1940. On the tree-area ratio and certain of its applications. J. Forest. 38: 311-317.

Daniel, T. W., J. A. Helms y F. S. Baker 1979. Principios de silvicultura. $2 d$ Ed. McGraw Hill. México. 490 pp.

Drew, T.J . y J . Flew elling. 1979. Stand density managment: An alternative approach and its application to Douglas Fir plantations. For. Sci. 25(3): 518-532.

Flew elling J.W. y T. Drew. 1985. Stand density management diagram for lodgepole pine. In: Bauwgarter R. Lodgepole pine: the species and its management. Coop. Ext. Washington.

García, E. 1982. Modificaciones al sistema de clasificación climática de Köppen. 3a. reimpresión. Instituto de Geografía, UNAM. México D.F $246 \mathrm{pp}$.

Gingrich, S.F. 1967. Measuring and evaluating stocking and stand density in upland hardwood forest in the Central States. Forest Science 13: 38-53.
Krajiceck, J. y Brinkman K. 1961. Crowncompetition: A measure of density. For. Sci. 7(1): 53-42.

Long N. J . 1985. A practical approach to density management. The Forestry Chonicle. February.

Martínez, M. 1992. Los Pinos Mexicanos. 3a. Ed. Ediciones Botas, México, D.F. $361 \mathrm{pp}$.

Márquez L., M. A. 1994. Unidades de vegetación de un bosque templado frío de San Dimas, Durango. Memorias del Simposio y II Reunión Nacional de Silvicultura y Manejo de Recursos Forestales. p. 14.

McCarter, J.B. y J.N. Long. 1986. A lodgepole pine density management diagram. Western J ournal of Applied Forestry 1: 6-11.

Reineke, L. H. 1933. Perfecting a stand density index for even-aged forest. J. Agr. Res. 46: 627-638.

Spurr, S. 1962. A measure of point density. For.Sci. 8(1): 85-96.

Westoby, M. 1984. The self-thinning rule. Adv.Ecol.Res. 14: 167-225.

Yoda, K., T. Kira, H. Ogawa y K. Hozumi. 1963. Intraespecific competition among higher plants. J our. Biol. Osaka City Univ. 14: 107-129.

Zar, J. H. 1984. Biostatistical Analysis. 2nd Ed. Prentice Hall, Englew ood Cliffs. N.J . 
Zepeda B., E.M. y M.E. Villareal D. 1987. Guía de densidad para Pinus hartwegii Lindl. de Zoquiapan, Méx. Div. Cien. For., U. A. Chapingo. 52 pp.
Weller D.E. 1989. The interespecific sizedensity relationship among crow dede plant stand and its implications for the $-3 / 2$ power rule of self-thinning. The American Naturalist 133(1): $20: 40$. 